\title{
Correction: Zarzycka, B. et al. Religious Struggle and Psychological Well-Being: The Mediating Role of Religious Support and Meaning Making. Religions 2020, 11, 149
}

\author{
Beata Zarzycka $^{1, *}\left(\mathbb{D}\right.$, Anna Tychmanowicz ${ }^{2}\left(\mathbb{D}\right.$ and Dariusz Krok ${ }^{3}(\mathbb{C}$ \\ 1 Institute of Psychology, The John Paul II Catholic University of Lublin, 20-950 Lublin, Poland \\ 2 Institute of Psychology, Maria Curie-Skłodowska University, 20-080 Lublin, Poland; \\ anna.tychmanowicz@poczta.umcs.lublin.pl \\ 3 Institute of Psychology, Opole University, 45-052 Opole, Poland; dkrok@uni.opole.pl \\ * Correspondence: zarzycka@kul.pl
}

Received: 23 April 2020; Accepted: 23 April 2020; Published: 28 April 2020

The authors want to make the following corrections to the paper (Zarzycka et al. 2020):

(1) Replacing Section 2.2.2:

2.2.2. Religious Support Scale (RSS)

The RSS consists of three seven-item scales, measuring the participant's perception of support from sources that are relevant to a religious context-God (e.g., God cares about your life and situation), congregation (e.g., you feel appreciated by the members of a congregation), and the clergy (e.g., you can turn to the church leadership for advice when you have problems). Each item was rated on a five-point Likert response scale from 1 (strongly disagree) to 5 (strongly agree). As such, higher scores on the RSS indicated a higher degree of feeling religiously supported (Fiala et al. 2002). In order to examine the factorial structure of the Polish RSS, we entered the 21 items into a principal component analysis (PCA) with direct oblimin rotation. This PCA, combined with the analysis of a scree plot, suggested a three-factor solution, explaining $73 \%$ of total variance. The internal consistency of the RSS obtained in the previous research ranged from $\alpha=0.75$ to 0.91 (Fiala et al. 2002) and in this study was $\alpha=0.91$ (Table 1).

With:

2.2.2. Polish Religious Support Scale (PRSS)

The PRSS (Zarzycka et al., forthcoming) is based on the Religious Support Scale (RSS; Fiala et al. 2002). It consists of three seven-item scales, measuring the participant's perception of support from sources that are relevant to a religious context-God (e.g., God cares about your life and situation), congregation (e.g., you feel appreciated by the members of a congregation), and the clergy (e.g., you can turn to the church leadership for advice when you have problems). The original RSS contained one reverse-scored item on each subscale to address response bias, but researchers (e.g., Lazar and Bjorck 2016; Yi and Bjorck 2014) have shown that this actually results in lower subscale reliabilities. Thus, all the items on the PRSS are rated on a five-point Likert response scale from 1 (strongly disagree) to 5 (strongly agree) with no reverse scoring. As such, higher scores on the PRSS indicate a higher degree of feeling religiously supported. The PRSS was translated and back-translated into Polish, and the factorial structure was assessed by entering the 21 items into a principal component analysis (PCA) with direct oblimin rotation. This PCA, combined with analysis of a scree plot, suggested the same three-factor solution, explaining 73\% of total variance. Fiala et al. reported the internal consistency of the RSS total score as 0.91 , and it was also 0.91 for the PRSS (Table 1).

(2) Adding Acknowledgments Section before References Section: 
Acknowledgments: The authors wish to acknowledge Jeffrey P. Bjorck, Ph.D., for his significant contributions to the development of the Polish Religious Support Scale.

(3) Adding Three New References:

Lazar, Aryeh, and Jeffrey P. Bjorck. 2016. Religious support and psychological well-being: Gender differences among religious Jewish Israelis. Mental Health, Religion, and Culture 19: 393-407. doi:10.1080/13674676.2016.1207160.

Yi, Gu Hwa, and Jeffrey P. Bjorck. 2014. Religious support and psychological functioning in Korean American Protestant Christians. Psychology of Religion and Spirituality 6: 44-52. doi:10.1037/a0034417.

Zarzycka, Beata, Anna Tychmanowicz, Tomasz Korulczyk, and Jeffrey P. Bjorck. Forthcoming. Religious Support and Psychological Functioning in a Polish Sample. manuscript in preparation.

The authors and the Editorial Office would like to apologize for any inconvenience caused to the readers by these changes. The changes do not affect the scientific results. The manuscript will be updated and the original will remain online on the article webpage.

\section{Reference}

Zarzycka, Beata, Anna Tychmanowicz, and Dariusz Krok. 2020. Religious Struggle and Psychological Well-Being: The Mediating Role of Religious Support and Meaning Making. Religions 11: 149. [CrossRef]

(C) 2020 by the authors. Licensee MDPI, Basel, Switzerland. This article is an open access article distributed under the terms and conditions of the Creative Commons Attribution (CC BY) license (http://creativecommons.org/licenses/by/4.0/). 